\title{
RAMIFICATION OVER HYPERSURFACES LOCATED IN SUBGENERAL POSITION OF THE GAUSS MAP OF COMPLETE MINIMAL SURFACES WITH FINITE TOTAL CURVATURE
}

\author{
Do Duc THAI and Pham Duc THOAN \\ (Received 14 May 2017 and revised 18 December 2017)
}

\begin{abstract}
The first aim of this paper is to show the second main theorem for holomorphic maps from a compact Riemann surface into the complex projective space which is ramified over hypersurfaces in subgeneral position. We then use it to study the ramification over hypersurfaces of the generalized Gauss map of complete regular minimal surfaces in $\mathbb{R}^{m}$ with finite total curvature, sharing hypersurfaces in subgeneral position. The results generalize our previous results [Thai and Thoan, Vietnam J. Math. 2017, doi:10.1007/s10013-017-0259-6].
\end{abstract}

\section{Introduction}

Value distribution theory of the Gauss map of complete regular minimal surfaces has a long history; in particular, much attention has been given to this theory from the viewpoint of the Nevanlinna theory. Over the last few decades, there have been several results on the ramification over hyperplanes of the generalized Gauss map of complete regular minimal surfaces in $\mathbb{R}^{m}$.

In 1991, M. Ru [7] showed an important theorem on the ramification over hyperplanes located in general position of the generalized Gauss map of complete regular minimal surfaces immersed in $\mathbb{R}^{m}$. In 2006, Y. Kawakami [5] found a non-flat complete minimal surface with finite total curvature in $\mathbb{R}^{3}$ such that the totally ramified value number of its Gauss map is $v=2.5$. In 2008, Y. Kawakami, R. Kobayashi and R. Miyaoka [6] obtained an estimate for the totally ramified value number of its Gauss map in terms of the degree of the Gauss map and the topological data of the surface.

As we know, when a minimal surface has finite total curvature, the surface is conformally equivalent to a compact Riemann surface (after the surface is equipped with a complex structure) punctured at a finite number of points and the (generalized) Gauss map is holomorphically extended to the compact Riemann surface.

For this reason, the minimal surfaces with finite total curvatures are called algebraic minimal surfaces and the theory of algebraic curves can be applied in this case. By an 2010 Mathematics Subject Classification: Primary 53A10; Secondary 53C42, 30D35, 32H30. Keywords: Gauss map of minimal surfaces; algebraic curves; second main theorem. The research of the authors is supported by an NAFOSTED grant of Vietnam (Grant No. 101.04-2017.317). 
algebraic curve, we mean a holomorphic map from a compact Riemann surface into the $n$ dimensional complex projective space $\mathbb{P}^{n}(\mathbb{C})$. For instance, in 2007, by using the Plücker formula, L. Jin and M. Ru [4] established the following theorem on the ramification over hyperplanes located in general position of the generalized Gauss map of the complete regular minimal surfaces immersed in $\mathbb{R}^{m}$ with finite total curvature.

TheOREM A. [4, Theorem 3.1] Let $x: S \rightarrow \mathbb{R}^{m}$ be a non-flat complete regular minimal surface with finite total curvature. Let $G: S \rightarrow \mathbb{P}^{m-1}(\mathbb{C})$ be its generalized Gauss map. Let $H_{1}, \ldots, H_{q}$ be hyperplanes in $P^{m-1}(\mathbb{C})$, located in general position in $P^{m-1}(\mathbb{C})$ $(1 \leq i \leq q)$. If $G$ is ramified over $H_{j}$ with multiplicity at least $m_{j}$ for each $j$ (note that if $G(M)$ omits $H_{j}$, then we take $\left.m_{j}=\infty\right)$, then

$$
\sum_{j=1}^{q}\left(1-\frac{m-1}{m_{j}}\right)<\frac{1}{2} m(m+1) .
$$

In particular, $G(S)$ can fail to intersect at most $m(m+1) / 2$ hyperplanes in general position in $\mathbb{P}^{m-1}(\mathbb{C})$.

Motivated by studying second main theorems for meromorphic mappings into the $n$ dimensional projective space sharing hypersurfaces in subgeneral position (see [1]), in the paper [8], we showed a second main theorem for algebraic curves into the $n$-dimensional projective space sharing hypersurfaces in subgeneral position. We then use it to study the value distribution of the generalized Gauss map of the complete (regular) minimal surfaces in $\mathbb{R}^{m}$ with finite total curvature, as well as the unicity problem, sharing hypersurfaces in subgeneral position. Unfortunately, the ramification problem over hypersurfaces located in subgeneral position of the generalized Gauss map of the complete regular minimal surfaces immersed in $\mathbb{R}^{m}$ with finite total curvature is not considered in the paper.

The main aim of this paper is to give an answer to the problem.

We now recall some notation. Let $M$ be a complete immersed minimal surface in $\mathbb{R}^{m}$. Take an immersion $x=\left(x_{0}, \ldots, x_{m-1}\right): M \rightarrow \mathbb{R}^{m}$. Then $M$ has the structure of a Riemann surface and any local isothermal coordinate $(x, y)$ of $M$ gives a local holomorphic coordinate $z=x+\sqrt{-1} y$. The generalized Gauss map of $x$ is defined to be

$$
G: M \rightarrow \mathbb{P}^{m-1}(\mathbb{C}), \quad G=\mathbb{P}\left(\frac{\partial x}{\partial z}\right)=\left(\frac{\partial x_{0}}{\partial z}: \cdots: \frac{\partial x_{m-1}}{\partial z}\right) .
$$

Since $x: M \rightarrow \mathbb{R}^{m}$ is immersed, it implies that

$$
g=g_{z}:=\left(g_{0}, \ldots, g_{m-1}\right)=\left(\left(g_{0}\right)_{z}, \ldots,\left(g_{m-1}\right)_{z}\right)=\left(\frac{\partial x_{0}}{\partial z}, \ldots, \frac{\partial x_{m-1}}{\partial z}\right)
$$

is a (local) reduced representation of $g$. Moreover, for another local holomorphic coordinate $\xi$ on $M$, we have $g_{\xi}=g_{z} \cdot(d z / d \xi)$ and hence $g$ is well defined (independently of the (local) holomorphic coordinate). Since $M$ is minimal, $G$ is a holomorphic map.

We now consider the hypersurface $Q$ given by

$$
\sum_{I \in \mathcal{I}_{d}} a_{I} z^{I}=0
$$

where

$$
\mathcal{I}_{d}=\left\{\left(i_{0}, \ldots, i_{n}\right) \in \mathbb{N}^{n+1}: i_{0}+\cdots+i_{n}=d\right\}, \quad I=\left(i_{0}, \ldots, i_{n}\right) \in \mathcal{I}_{d},
$$


$z^{I}=z_{0}^{i_{0}} \cdots z_{n}^{i_{n}}$ and $a_{I} \in \mathbb{C}\left(I \in \mathcal{I}_{d}\right)$. Put

$$
M=\left(\begin{array}{c}
n+d \\
n
\end{array}\right)-1
$$

and denote by

$$
H=\left\{\left(z_{0}, \ldots, z_{M}\right) \in \mathbb{C}^{M+1}: \sum_{I_{j} \in \mathcal{I}_{d}} a_{I_{j}} z_{I_{j}}=0\right\}
$$

the hyperplane in $\mathbb{C}^{M+1}$ associated with $Q_{i}$.

Let $f: S \rightarrow \mathbb{P}^{n}(\mathbb{C})$ be a holomorphic map with a reduced (local) representation $f(z)=$ $\left(f_{0}(z), \ldots, f_{n}(z)\right)$. For each $d$, define $F: S \rightarrow \mathbb{P}^{M}(\mathbb{C})$ by

$$
F(z)=\left(f^{I_{0}}(z), \ldots, f^{I_{M}}(z)\right),
$$

where $\left\{I_{0}, \ldots, I_{M}\right\}=\mathcal{I}_{d}$ and $f^{I}(z)=f_{0}^{i_{0}}(z) \cdots f_{n}^{i_{n}}(z)$ for $I=\left(i_{0}, \ldots, i_{n}\right) \in \mathcal{I}_{d}$. Such definition is independent of the choice of the representation of $f$ and of the parameter $z$. We call $F$ the associated map with $f$ of degree $d$. Put $Q(f)=H(F)=\sum_{I \in \mathcal{I}_{d}} a_{I} f^{I}$. We will consider $f^{*} Q=v_{Q(f)}$ as a divisor.

Definition 1. The map $f$ is said to be ramified over a hypersurface $Q$ in $\mathbb{P}^{m-1}(\mathbb{C})$ with multiplicity at least $e$ if all the zeros of the function $Q(f)$ have orders at least $e$.

If the image of $f$ omits $Q$, one will say that $f$ is ramified over $Q$ with multiplicity $\infty$.

Definition 2. Let $\left\{Q_{j}\right\}_{j=1}^{q}$ be the hypersurfaces in $\mathbb{P}^{n}(\mathbb{C})$. Denote by $Q$ the index set $\{1, \ldots, q\}$. Let $N \geq n$ and $q \geq N+1$. The family $\left\{Q_{j}\right\}_{j=1}^{q}$ is said to be in $N$-subgeneral position in $\mathbb{P}^{n}(\mathbb{C})$ if for every subset $R \subset Q$ with the cardinality $|R|=N+1$, then

$$
\bigcap_{j \in R} Q_{j}=\emptyset .
$$

If they are in $n$-subgeneral position, we simply say that they are in general position.

We now prove the following.

THEOREM 1. Let $S$ be a compact complex Riemann surface of genus $g$. Let $f: S \rightarrow$ $\mathbb{P}^{n}(\mathbb{C})$ be a holomorphic curve such that $f(S) \subset \mathbb{P}^{k}(\mathbb{C})$ and $f(S)$ is not contained in any hypersurface in $\mathbb{P}^{k}(\mathbb{C})$ for some $1 \leq k \leq n$. Let $Q_{1}, \ldots, Q_{q}$ be the hypersurfaces in $\mathbb{P}^{n}(\mathbb{C})$, located in $N$-subgeneral position with $d_{i}:=\operatorname{deg} Q_{i}(1 \leq i \leq q)$. Put $d=\operatorname{lcm}\left(d_{1}, \ldots, d_{q}\right)$ and

$$
M=\left(\begin{array}{c}
k+d \\
k
\end{array}\right)-1
$$

Let $E$ be a finite subset of $S$. Then

$$
\begin{aligned}
(q & \left.-\frac{(2 N-k+1)(M+1)}{k+1}\right) \operatorname{deg}(f) \\
& \leq \sum_{j=1}^{q} \sum_{P \notin E} \frac{1}{d_{j}} \min \left\{M, v_{Q_{j}(f)}(P)\right\}+\frac{(2 N-k+1) M(M+1)}{2(k+1)} \cdot \frac{2(g-1)+|E|}{d},
\end{aligned}
$$

where $v_{Q_{j}(f)}=f^{*} Q_{j}(1 \leq j \leq q)$ is the vanishing order of $Q(f)$. 
THEOREM 2. Let $x: S \rightarrow \mathbb{R}^{m}$ be a non-flat complete regular minimal surface with finite total curvature. Let $G: S \rightarrow \mathbb{P}^{m-1}(\mathbb{C})$ be its generalized Gauss map. Assume that $G(S) \subset \mathbb{P}^{k}(\mathbb{C})$ and $G(S)$ is not contained in any hypersurface in $\mathbb{P}^{k}(\mathbb{C})$ for some $1 \leq k \leq m-1$. Let $Q_{1}, \ldots, Q_{q}$ be hypersurfaces in $\mathbb{P}^{m-1}(\mathbb{C})$, located in $N$-subgeneral position in $\mathbb{P}^{m-1}(\mathbb{C})$ with $\operatorname{deg} Q_{i}=d_{i}(1 \leq i \leq q)$. Let $d=\operatorname{lcm}\left(d_{1}, \ldots, d_{q}\right)$. Assume that $G$ is ramified over hypersurfaces $Q_{j}$ with multiplicity at least $m_{j}$ for each $j$ and

$$
M_{k}=\left(\begin{array}{c}
k+d \\
k
\end{array}\right)-1
$$

Then

$$
\sum_{j=1}^{q}\left(1-\frac{M_{k}}{m_{j}}\right)<\frac{(2 N-k+1)\left(M_{k}+1\right)\left(M_{k}+2 d\right)}{2(k+1) d} .
$$

In particular, for each $1 \leq k \leq m-1$, then

$$
\sum_{j=1}^{q}\left(1-\frac{m-1}{m_{j}}\right)<\frac{(2 N-m+2)(m+1)}{2}
$$

if $d=1$ and

$$
\sum_{j=1}^{q}\left(1-\frac{M}{m_{j}}\right)<\frac{(2 N-m+2)(M+1)(M+2 d)}{2 d m}
$$

if $d>1$, where

$$
M=\left(\begin{array}{c}
m-1+d \\
m-1
\end{array}\right)-1
$$

\section{Auxiliary lemmas}

We now recall auxiliary results of Nochka weights for hypersurfaces in subgeneral position and the theory of algebraic curves in the projective spaces which will be used later.

Let $\left\{H_{i}\right\}_{i=1}^{q}$ be $q$ hyperplanes in $\mathbb{C}^{M+1}$ passing through the origin of coordinates. Assume that each $H_{i}$ is defined by the linear equation

$$
a_{i 0} z_{0}+\cdots+a_{i M} z_{M}=0,
$$

where $a_{i j} \in \mathbb{C}(j=0, \ldots, M)$, not all zeros. We define the vector associated with $H_{i}$ by

$$
v_{i}=\left(a_{i 0}, \ldots, a_{i M}\right) \in \mathbb{C}^{M+1} .
$$

For each subset $R \subset\{1, \ldots, q\}$, the rank of $\left\{H_{i}\right\}_{i \in R}$ is defined by

$$
\operatorname{rank}\left\{H_{i}\right\}_{i \in R}=\operatorname{rank}\left\{v_{i}\right\}_{i \in R} .
$$

The family $\left\{H_{i}\right\}_{i=1}^{q}$ is said to be in $N$-subgeneral position if, for any subset $R \subset\{1, \ldots, q\}$ with $\sharp R=N+1, \bigcap_{i \in R} H_{i}=\{0\}$, i.e., $\operatorname{rank}\left\{H_{i}\right\}_{i \in R}=M+1$.

Similar to [1, Lemma 4.2], we have the following.

LeMmA 3. Let $\left\{H_{i}\right\}_{i=1}^{q}$ be a set of hyperplanes in $\mathbb{P}^{M}(\mathbb{C})$. Then, there exist $(M-n)$ hyperplanes $\left\{U_{i}\right\}_{i=1}^{M-n}$ such that $\operatorname{rank}\left\{\left\{H_{i}\right\}_{i \in R} \cup\left\{U_{i}\right\}_{i=1}^{M-n}\right\}=M+1$ for any subset $R \subset$ $\{1, \ldots, q\}$ with $|R|=\operatorname{rank}\left\{H_{i}\right\}_{i \in R}=n+1$. 
Proof. For each subset $R \subset\{1, \ldots, q\}$ with $|R|=\operatorname{rank}\left\{H_{i}\right\}_{i \in R}=n+1$, denote by $V_{R}$ the set of all vectors $v=\left(v_{1}, \ldots, v_{M-n}\right) \in\left(\mathbb{C}^{M+1}\right)^{M-n}$ such that $\left\{\left\{H_{i}\right\}_{i \in R}, v_{1}, \ldots, v_{M-n}\right\}$ is linearly dependent over $\mathbb{C}$. Since $\operatorname{dim} \mathbb{C}^{M+1}=M+1$ and $\operatorname{rank}\left\{H_{i}\right\}_{i \in R}=n+1$, there exists a vector $u=\left(u_{1}, \ldots, u_{M-n}\right) \in\left(\mathbb{C}^{M+1}\right)^{M-n}$ such that $\left\{\left\{H_{i}\right\}_{i \in R}, u_{1}, \ldots, u_{M-n}\right\}$ is linearly independent over $\mathbb{C}$, i.e., $u \notin V_{R}$. Therefore $V_{R}$ is a proper algebraic subset of $\left(\mathbb{C}^{M+1}\right)^{M-n}$ for each $R$. This implies that

$$
\left(\mathbb{C}^{M+1}\right)^{M-n} \backslash \bigcup_{R} V_{R} \neq \emptyset
$$

Hence, there is a vector $u^{*}=\left(u_{1}^{*}, \ldots, u_{M-n}^{*}\right) \in\left(\mathbb{C}^{M+1}\right)^{M-n} \backslash \bigcup_{R} V_{R} \neq \emptyset$. Take a hyperplane $H_{i}(1 \leq i \leq M-n)$ in $\mathbb{P}^{N}(\mathbb{C})$, which is defined by $u_{i}^{*}$. We have

$$
\operatorname{rank}\left\{\left\{H_{i}\right\}_{i \in R} \cup\left\{U_{i}\right\}_{i=1}^{M-n}\right\}=M+1
$$

for every subset $R \subset\{1, \ldots, q\}$ with $|R|=\operatorname{rank}\left\{H_{i}\right\}_{i \in R}=n+1$. The lemma is proved.

Let $\left\{Q_{i}\right\}_{i=1}^{q}$ be $q$ hypersurfaces in $\mathbb{P}^{n}(\mathbb{C})$ of the common degree $d$. Assume that each $Q_{i}$ is defined by a homogeneous polynomial $Q_{i}^{*} \in \mathbb{C}\left[x_{0}, \ldots, x_{n}\right]$. We regard $\mathbb{C}\left[x_{0}, \ldots, x_{n}\right]$ as a complex vector space and define

$$
\operatorname{rank}\left\{Q_{i}\right\}_{i \in R}=\operatorname{rank}\left\{Q_{i}^{*}\right\}_{i \in R}
$$

for every subset $R \subset\{1, \ldots, q\}$. It is easy to see that

$$
\operatorname{rank}\left\{Q_{i}\right\}_{i \in R}=\operatorname{rank}\left\{Q_{i}^{*}\right\}_{i \in R} \geq n+1-\operatorname{dim}\left(\bigcap_{i \in R} Q_{i}\right) .
$$

Hence, if $\left\{Q_{i}\right\}_{i=1}^{q}$ is in $N$-subgeneral position, by the above equality, we have

$$
\operatorname{rank}\left\{Q_{i}\right\}_{i \in R} \geq n+1
$$

for any subset $R \subset\{1, \ldots, q\}$ with $\sharp R=N+1$. Moreover, assume that each $Q_{i}$ is given by

$$
\sum_{I \in \mathcal{I}_{d}} a_{i I} z^{I}=0
$$

where

$$
\begin{gathered}
\mathcal{I}_{d}=\left\{\left(i_{0}, \ldots, i_{n}\right) \in \mathbb{N}^{n+1}: i_{0}+\cdots+i_{n}=d\right\}, \quad I=\left(i_{0}, \ldots, i_{n}\right) \in \mathcal{I}_{d}, \\
z^{I}=z_{0}^{i_{0}} \cdots z_{n}^{i_{n}} \text { and } a_{i I} \in \mathbb{C}\left(1 \leq i \leq q, I \in \mathcal{I}_{d}\right) . \text { Put } \\
M=\left(\begin{array}{c}
n+d \\
n
\end{array}\right)-1
\end{gathered}
$$

and denote by

$$
H_{i}=\left\{\left(z_{i_{0}}, \ldots, z_{i_{M}}\right) \in \mathbb{C}^{M+1}: \sum_{I_{j} \in \mathcal{I}_{d}} a_{i I_{j}} z_{I_{j}}=0\right\}
$$

the hyperplane in $\mathbb{C}^{M+1}$ associated with $Q_{i}$. It is easy to see that $\operatorname{rank}\left\{Q_{i}\right\}_{i \in R}=\operatorname{rank}\left\{H_{i}\right\}_{i \in R}$.

By [1, Lemma 3.3], we have the following. 
Lemma 4. [1, Lemma 3.3] Let $Q_{1}, \ldots, Q_{q}(q>2 N-n+1)$ be hypersurfaces of the common degree $d$ in $\mathbb{P}^{n}(\mathbb{C})$ located in $N$-subgeneral position. Then there exists a function $\omega:\{1, \ldots, q\} \rightarrow(0,1]$ called a Nochka weight and a real number $\theta \geq 1$ called a Nochka constant satisfying the following conditions.

(i) If $j \in\{1, \ldots, q\}$, then $0<\omega(j) \theta \leq 1$.

(ii) $\quad q-2 N+n-1=\theta\left(\sum_{j=1}^{q} \omega(j)-n-1\right)$.

(iii) For $R \subset\{1, \ldots, q\}$ with $|R|=N+1$, then $\sum_{i \in R} \omega(i) \leq n+1$.

(iv) $(N+1) /(n+1) \leq \theta \leq(2 N-n+1) /(n+1)$.

(v) Given real numbers $\lambda_{1}, \ldots, \lambda_{q}$ with $\lambda_{j} \geq 1$ for $1 \leq j \leq q$ and given any $R \subset$ $\{1, \ldots, q\}$ and $|R|=N+1$, there exists a subset $R^{o} \subset R$ such that $\left|R^{0}\right|=$ $\operatorname{rank}\left\{Q_{i}\right\}_{i \in R^{0}}=n+1$ and

$$
\prod_{i \in R} \lambda_{i}^{\omega(i)} \leq \prod_{i \in R^{0}} \lambda_{i}
$$

Assume that $f: S \rightarrow \mathbb{P}^{n}(\mathbb{C})$ is a linearly non-degenerate algebraic curve (that is, $f(S)$ is not contained in any hyperplane in $\mathbb{P}^{n}(\mathbb{C})$ ). For every point $P \in S$, in a neighborhood of $P$, let $\mathbf{f}(z)=\left(f_{0}(z), \ldots, f_{n}(z)\right)$ be a reduced representation of $f$ at $P$ with $z(P)=0$, where $z$ is a local parameter for $S$ at $P$ and $f_{0}, \ldots, f_{n}$ are holomorphic functions without common zeros. Take a hyperplane $H: a_{0} z_{0}+\cdots+a_{n} z_{n}=0$ in $\mathbb{P}^{n}(\mathbb{C})$ and put

$$
H(f)=a_{0} f_{0}+\cdots+a_{n} f_{n} .
$$

Then $\sum_{z \in S} v_{H(f)}(z)$ does not depend on the choice of $H$, where $v_{H(f)}(z)$ is the intersection multiplicity of the images of $f$ and $H$ at $f(z)$. We define the degree of $f$ by

$$
\operatorname{deg}(f)=\sum_{P \in S} v_{H(f)}(P) .
$$

It is easy to see that if $f^{-1}(H)=\left\{P_{1}, \ldots, P_{r}\right\}$, then

$$
\operatorname{deg}(f)=\sum_{j=1}^{r} v_{H(f)}\left(P_{j}\right) \geq r .
$$

Now we may assume that $f(0)=(1,0, \ldots, 0)$ by making a linear change of coordinates in $\mathbb{C}^{n+1}$. We have $f_{1}(0)=\cdots=f_{n}(0)=0$. Write $\left(f_{1}(z), \ldots, f_{n}(z)\right)=$ $z^{\delta_{1}}\left(f_{1}^{1}(z), \ldots, f_{n}^{1}(z)\right)$ with $\left(f_{1}^{1}(0), \ldots, f_{n}^{1}(0)\right) \neq 0$. Make a linear change of the last $n$ coordinate $\mathbb{C}^{n+1}$ so that $\left(f_{1}^{1}(0), \ldots, f_{n}^{1}(0)\right)=(1,0, \ldots, 0)$. Write $\left(f_{2}^{1}(z), \ldots, f_{n}^{1}(z)\right)=$ $z^{\delta_{2}-\delta_{1}}\left(f_{2}^{2}(z), \ldots, f_{n}^{2}(z)\right)$ with $\left(f_{2}^{2}(0), \ldots, f_{n}^{2}(0)\right) \neq 0$. Continuing in this way we end up with a system of coordinates for $\mathbb{C}^{n+1}$ in terms of which

$$
f(z)=\left(z^{\delta_{0}}+\cdots, z^{\delta_{1}}+\cdots, \ldots, z^{\delta_{n}}+\cdots\right),
$$

where $0=\delta_{0}<\delta_{1}<\cdots<\delta_{n}$. Put $v_{i}=\delta_{i+1}-\delta_{i}-1,0 \leq i \leq n-1$ and note that, for $P \in$ $S$, we have

$$
\sum_{i=0}^{n}(n-i) v_{i}(P)+\frac{1}{2} n(n+1)=\delta_{0}(P)+\delta_{1}(P)+\cdots+\delta_{n}(P) .
$$

Let

$$
\sigma_{i}=\sum_{P \in S} v_{i}(P)
$$


By the Plücker formula, which is a generalization of the Riemann-Hurwitz theorem (see [3]), we have

$$
\sum_{i=0}^{n}(n-i) \sigma_{i}=(n+1) \operatorname{deg}(f)+n(n+1)(g-1) .
$$

Here $g$ stands for the genus of $S$.

We need the following.

Lemma 5. Let $f: S \rightarrow \mathbb{P}^{n}(\mathbb{C})$ be an algebraic curve and $F: S \rightarrow \mathbb{P}^{M}(\mathbb{C})$ be the associated map with $f$ of degree $d$. Let $Q$ be a hypersurface in $\mathbb{P}^{n}(\mathbb{C})$ of degree $d$. Then

$$
\operatorname{deg}(F)=\sum_{P \in S} v_{Q(f)}(P)=d \operatorname{deg}(f) .
$$

Proof. Let $H=\left\{\omega_{0}=0\right\}$ be a hyperplane in $\mathbb{P}^{M}(\mathbb{C})$. Assume that $F^{-1}(H)=\left\{P_{1}, \ldots, P_{r}\right\}$. For each $1 \leq j \leq r$, take a holomorphic local parameter $z_{j}$ with $z_{j}\left(P_{j}\right)=0$ in a neighborhood of $P_{j}$ in $S$. Consider a sufficiently small positive $\epsilon$ such that $\bar{U}_{j}(\epsilon):=\left\{z_{j}:\left|z_{j}\right| \leq \epsilon\right\}$ are mutually disjoint. Now take a reduced representation $f(z)=\left(f_{0}(z), \ldots, f_{n}(z)\right)$ of $f$ on $\bigcup_{j} U_{j}(\epsilon)$. We obtain $H(F)(z)=f^{I_{0}}(z)=\left(f_{0}(z)\right)^{d}$, where $I_{0}=(1,0, \ldots, 0) \in \mathcal{I}_{d}$. This implies that

$$
\begin{aligned}
\operatorname{deg}(F) & =\sum_{j=1}^{r} v_{H(F)}\left(P_{j}\right)=\sum_{j=1}^{r} v_{f_{0}^{d}}\left(P_{j}\right)=\sum_{j=1}^{r} d \cdot v_{f_{0}}\left(P_{j}\right) \\
& =\sum_{j=1}^{r} d \cdot v_{\hat{H}(f)}\left(P_{j}\right)=d \operatorname{deg}(f),
\end{aligned}
$$

where $\hat{H}: \omega_{0}=0$ is a hyperplane in $\mathbb{P}^{n}(\mathbb{C})$. By taking the associated hyperplane $U$ of $Q$, we have

$$
\operatorname{deg}(F)=\sum_{P \in S} v_{U(F)}(P)=\sum_{P \in S} v_{Q(f)}(P) .
$$

The proof of Lemma 5 is complete.

\section{The proof of Theorem 1}

We consider two cases.

Case 1: the map $f: S \rightarrow \mathbb{P}^{n}(\mathbb{C})$ is a holomorphic curve such that $f(S)$ is not contained in any hypersurface in $\mathbb{P}^{n}(\mathbb{C})$

Step 1. We first prove the theorem in the case where all hypersurfaces $Q_{i}(i=1, \ldots, q)$ have the same degree $d$. Since $f$ is algebraically non-degenerate, $F$ is a linearly non-degenerate algebraic curve of $S$ into $\mathbb{P}^{M}(\mathbb{C})$, where

$$
M=\left(\begin{array}{c}
n+d \\
n
\end{array}\right)-1
$$

(i.e., its image is not contained in any hyperplanes in $\mathbb{P}^{M}(\mathbb{C})$ ). 
Denote by $H_{i}$ the associated hyperplane of hypersurface $Q_{i} \quad(1 \leq i \leq q)$. By Lemma 3, we can choose the hyperplanes $\left\{U_{i}\right\}_{i=0}^{M-n-1}$ in $\mathbb{P}^{M}(\mathbb{C})$ such that $\operatorname{rank}\left\{\left\{H_{i}\right\}_{i \in R} \cup\right.$ $\left.\left\{U_{i}\right\}_{i=0}^{M-n-1}\right\}=M+1$ for any subset $R \subset\{1, \ldots, q\}$ with $|R|=\operatorname{rank}\left\{H_{i}\right\}_{i \in R}=n+1$.

Consider the point $P \in E$. Since $\left\{Q_{j}\right\}_{j=1}^{q}$ is in $N$-subgeneral position, there exist at most $N$ hypersurfaces which can intersect $F(S)$ at $P$. Without loss of generality, we may assume that $F(S)$ intersects $Q_{j}(1 \leq j \leq N)$ and $F(S)$ does not intersect $Q_{j}$ with $j>N$. Put $R=\{1, \ldots, N+1\}$ and choose $R^{0} \subset R$ with $\left|R^{0}\right|=\operatorname{rank}\left\{Q_{j}\right\}_{j \in R^{0}}=n+1$ such that $R^{0}$ satisfies Lemma 4(v) with respect to numbers $\lambda_{j}=e^{v_{Q_{j}(f)}(P)}$. Then, we have

$$
\prod_{j \in R} e^{\omega(j) v_{Q_{j}(f)}(P)} \leq \prod_{j \in R^{0}} e^{v_{Q_{j}(f)}(P)},
$$

where $\omega(j)$ are the Nochka weights associated to the hypersurfaces $Q_{j}(1 \leq j \leq q)$. This implies that

$$
\sum_{j=1}^{q} \omega(j) v_{Q_{j}(f)}(P)=\sum_{j \in R} \omega(j) v_{Q_{j}(f)}(P) \leq \sum_{j \in R_{0}} v_{Q_{j}(f)}(P) .
$$

For the linearly independent family of hyperplanes $\left\{\left\{H_{j}\right\}_{j \in R^{0}},\left\{U_{i}\right\}_{i=0}^{M-n-1}\right\}$, take a local parameter $z$ for $S$ at $P$ such that $z(P)=0$ and write $F$ in the form in (2.2). At $P$ the maximum possible value of $v_{Q_{j}(f)}(P)=v_{\left(F, H_{j}\right)}(P)\left(j \in R^{0}\right)$ or $v_{U_{i}(F)}(P)(0 \leq i \leq M-n-1)$ is $\delta_{M}(P)$, and for the unique hyperplane $z_{M}=0$. A second hyperplane can intersect $F(S)$ at $P$ with multiplicities at most $\delta_{M-1}(P), \ldots$ It follows that

$$
\sum_{i=0}^{M-n-1} v_{\left(F, U_{i}\right)}(P)+\sum_{j} v_{Q_{j}(f)}(P) \leq \delta_{0}(P)+\delta_{1}(P)+\cdots+\delta_{M}(P) .
$$

By (2.3), we have

$$
\sum_{i=0}^{M-n-1} v_{\left(F, U_{i}\right)}(P)+\sum_{j \in R_{0}} v_{Q_{j}(f)}(P) \leq \sum_{i=0}^{M}(M-i) v_{i}(P)+\frac{1}{2} M(M+1) .
$$

Combining (3.2) with (3.1), we get

$$
\sum_{j=1}^{q} \omega(j) v_{Q_{j}(f)}(P) \leq \sum_{i=0}^{M}(M-i) v_{i}(P)+\frac{1}{2} M(M+1)-\sum_{i=0}^{M-n-1} v_{U_{i}(F)}(P) .
$$

Hence, we have

$$
\sum_{i=0}^{M} \sum_{P \in E}(M-i) v_{i}(P) \geq \sum_{j=1}^{q} \sum_{P \in E} \omega(j) v_{Q_{j}(f)}(P)-\frac{1}{2} M(M+1)|E| .
$$

Consider the point $P \notin E$. There exist at most $N$ hypersurfaces which can intersect $F(S)$ at $P$. We may assume that $F(S)$ intersects $Q_{j}, j \in A$, with $|A|=N$ and $F(S)$ does not intersect $Q_{j}$, with $j \notin A$. Take $R_{1} \subset\{1, \ldots, q\}$ such that $R_{1} \supset A$ and $\left|R_{1}\right|=N+1$. We choose $R_{1}^{0} \subset R_{1}$ with $\left|R_{1}^{0}\right|=\operatorname{rank}\left\{Q_{j}\right\}_{j \in R_{1}^{0}}=n+1$ such that $R_{1}^{0}$ satisfies Lemma 4(v) with respect to numbers $\lambda_{j}=e^{v_{Q_{j}(f)}(P)-\min \left\{M, v_{Q_{j}(f)}(P)\right\}}(1 \leq j \leq q)$. Then, we have

$$
\prod_{j \in R_{1}} e^{\omega(j) v_{Q_{j}(f)}(P)\left[v_{Q_{j}(f)}(P)-\min \left\{M, v_{Q_{j}(f)}(P)\right\}\right]} \leq \prod_{j \in R_{1}^{0}} e^{v_{Q_{j}(f)}(P)-\min \left\{M, v_{Q_{j}(f)}(P)\right\}} .
$$


This gives

$$
\begin{aligned}
\sum_{j=1}^{q} \omega(j)\left[v_{Q_{j}(f)}(P)-\min \left\{M, v_{Q_{j}(f)}(P)\right\}\right] & =\sum_{j \in R_{1}} \omega(j)\left[v_{Q_{j}(f)}(P)-\min \left\{M, v_{Q_{j}(f)}(P)\right\}\right] \\
& \leq \sum_{j \in R_{1}^{0}}\left[v_{Q_{j}(f)}(P)-\min \left\{M, v_{Q_{j}(f)}(P)\right\}\right] .
\end{aligned}
$$

Denote $n+1$ hypersurfaces $Q_{j}\left(j \in R_{1}^{0}\right)$ by $Q_{P, M-n}, \ldots, Q_{P, M}$ and we have the linearly independent family of hyperplanes $\left\{\left\{H_{P, j}\right\}_{j=M-n}^{M},\left\{U_{i}\right\}_{i=0}^{M-n-1}\right\}$. Without loss of generality, we assume that

$$
v_{\left(F, U_{0}\right)}(P) \leq v_{\left(F, U_{1}\right)}(P) \leq \cdots \leq v_{\left(F, U_{M-n-1}\right)}(P) \leq v_{Q_{P, M-n}(f)}(P) \leq \cdots \leq v_{Q_{P, M}(f)}(P) .
$$

Then, $v_{\left(F, U_{i}\right)}(P) \leq \delta_{i}(P)(0 \leq i \leq M-n-1)$ and $v_{Q_{P, M-n+j}(f)}(P) \leq \delta_{M-n+j}(P)(0 \leq$ $j \leq n)$. Assume that $v_{Q_{P, M-n+j}(f)}(P) \leq M$ for $0 \leq j<k_{0}$, and $v_{Q_{P, M-n+j}(f)}(P)>M$ for $k_{0} \leq j \leq n$, where $0 \leq k_{0} \leq n+1$. Then

$$
\sum_{j=0}^{n}\left[v_{Q_{P, M-n+j}(f)}(P)-\min \left\{M, v_{Q_{P, M-n+j}(f)}(P)\right\}\right]=\sum_{j=k_{0}}^{n}\left[v_{Q_{P, M-n+j}(f)}(P)-M\right] .
$$

On the other hand, since $\delta_{i} \geq i$ for $0 \leq i \leq M$ and $v_{Q_{P, M-k+j}(f)}(P) \leq \delta_{M-k+j}(P)$ for $0 \leq$ $j \leq n$, we have

$$
\begin{aligned}
\sum_{j=0}^{n}\left[\delta_{M-n+j}(P)-(M-n+j)\right] & \geq \sum_{j=k_{0}}^{n}\left[\delta_{M-n+j}(P)-(M-n+j)\right] \\
& \geq \sum_{j=k_{0}}^{n}\left[\delta_{M-n+j}(P)-M\right] \\
& \geq \sum_{j=k_{0}}^{n}\left[v_{Q_{P, M-n+j}(f)}(P)-M\right] .
\end{aligned}
$$

Combining (2.3) with (3.4)-(3.6), we get

$$
\begin{aligned}
\sum_{i=0}^{M}(M-i) v_{i}(P) & =\sum_{i=0}^{M}\left(\delta_{i}(P)-i\right) \\
& \geq \sum_{j=0}^{n}\left[\delta_{M-n+j}(P)-(M-n+j)\right] \\
& \geq \sum_{j=1}^{q} \omega(j)\left[v_{Q_{j}(f)}(P)-\min \left\{M, v_{Q_{j}(f)}(P)\right\}\right] .
\end{aligned}
$$

Therefore,

$$
\sum_{i=0}^{M} \sum_{P \notin E}(M-i) v_{i}(P) \geq \sum_{j=1}^{q} \sum_{P \notin E} \omega(j) v_{Q_{j}(f)}(P)-\sum_{j=1}^{q} \sum_{P \notin E} \omega(j) \min \left\{M, v_{Q_{j}(f)}(P)\right\} .
$$


From (3.3) and by the above inequality, we get

$$
\begin{aligned}
\sum_{i=0}^{M} \sum_{P \in S}(M-i) v_{i}(P) \geq & \sum_{j=1}^{q} \sum_{P \in S} \omega(j) v_{Q_{j}(f)}(P)-\sum_{j=1}^{q} \sum_{P \notin E} \omega(j) \min \left\{M, v_{Q_{j}(f)}(P)\right\} \\
& -\frac{1}{2} M(M+1)|E| .
\end{aligned}
$$

Combining this inequality with (2.4) and (2.5), we get

$$
\begin{aligned}
& (M+1) \operatorname{deg}(F)+M(M+1)(g-1) \\
& \geq \sum_{j=1}^{q} \sum_{P \in S} \omega(j) v_{Q_{j}(f)}(P)-\sum_{j=1}^{q} \sum_{P \notin E} \omega(j) \min \left\{M, v_{Q_{j}(f)}(P)\right\}-\frac{1}{2} M(M+1)|E| .
\end{aligned}
$$

Hence,

$$
\begin{aligned}
& \sum_{j=1}^{q} \sum_{P \in S} \omega(j) v_{Q_{j}(f)}(P)-(M+1) \operatorname{deg}(F) \\
& \quad \leq \sum_{j=1}^{q} \sum_{P \notin E} \omega(j) \min \left\{M, v_{Q_{j}(f)}(P)\right\}+\frac{1}{2} M(M+1)\{2(g-1)+|E|\} .
\end{aligned}
$$

By Lemma 5, this inequality implies that

$$
\begin{aligned}
& \sum_{j=1}^{q}(\omega(j)-M-1) d \operatorname{deg}(f) \\
& \quad \leq \sum_{j=1}^{q} \sum_{P \notin E} \omega(j) \min \left\{M, v_{Q_{j}(f)}(P)\right\}+\frac{1}{2} M(M+1)\{2(g-1)+|E|\} .
\end{aligned}
$$

Using Lemma 4(i), we get

$$
\begin{aligned}
\left(\sum_{j=1}^{q} \omega(j)-M-1\right) \theta & =\left(\sum_{j=1}^{q} \omega(j)-n-1-(M-n)\right) \theta \\
& =\left(\frac{q-2 N+n-1}{\theta}-(M-n)\right) \theta \\
& =(q-2 N+n-1)-(M-n) \theta .
\end{aligned}
$$

Combining this inequality with (3.9), we have

$$
\begin{aligned}
& ((q-2 N+n-1)-(M-n) \theta) d \operatorname{deg}(f) \\
& \quad \leq \sum_{j=1}^{q} \sum_{P \notin E} \theta \omega(j) \min \left\{M, v_{Q_{j}(f)}(P)\right\}+\frac{1}{2} M(M+1)\{2(g-1)+|E|\} \theta .
\end{aligned}
$$

It follows from Lemma 4(i) and (iv) that

$$
\begin{aligned}
(q & \left.-\frac{(2 N-n+1)(M+1)}{n+1}\right) \operatorname{deg}(f) \\
& \leq \frac{1}{d} \sum_{j=1}^{q} \sum_{P \notin E} \min \left\{M, v_{Q_{j}(f)}(P)\right\}+\frac{(2 N-n+1) M(M+1)}{2(n+1)} \cdot \frac{2(g-1)+|E|}{d} .
\end{aligned}
$$

Hence, the theorem is proved in the case where all $Q_{i}$ have the same degree. 
Step 2. We now prove the theorem in the general case where deg $Q_{i}=d_{i}(i=1, \ldots, q)$. We put $T_{i}=Q_{i}^{d / d_{i}}(1 \leq i \leq q)$. It is easy to see that the hypersurfaces $T_{1}, \ldots, T_{q}$ have the same degree $d$ and they are still in $N$-subgeneral position in $\mathbb{P}^{n}(\mathbb{C})$. Similar to (3.8) in Step 1, we have

$$
\begin{aligned}
& \sum_{j=1}^{q} \sum_{P \in S} \omega(j) v_{Q_{j}^{d / d_{j}}(f)}(P)-(M+1) \operatorname{deg}(F) \\
& \quad \leq \sum_{j=1}^{q} \sum_{P \notin E} \omega(j) \min \left\{M, v_{Q_{j}^{d / d_{j}}(f)}(P)\right\}+\frac{1}{2} M(M+1)\{2(g-1)+|E|\} .
\end{aligned}
$$

Therefore, we get

$$
\begin{aligned}
& \sum_{j=1}^{q} \omega(j) \frac{d}{d_{j}} \sum_{P \in E} v_{Q_{j}(f)}(P)-(M+1) \operatorname{deg}(F) \\
& \quad \leq \sum_{j=1}^{q} \sum_{P \notin E} \omega(j) \frac{d}{d_{j}} \min \left\{M, v_{Q_{j}(f)}(P)\right\}+\frac{1}{2} M(M+1)\{2(g-1)+|E|\} .
\end{aligned}
$$

By Lemma 5 and (2.6), this inequality implies that

$$
\begin{aligned}
& \sum_{j=1}^{q} \omega(j) \frac{d}{d_{j}} d_{j} \operatorname{deg}(f)-(M+1) d \operatorname{deg}(f) \\
& \quad \leq \sum_{j=1}^{q} \sum_{P \notin E} \omega(j) \frac{d}{d_{j}} \min \left\{M, v_{Q_{j}(f)}(P)\right\}+\frac{1}{2} M(M+1)\{2(g-1)+|E|\} .
\end{aligned}
$$

Hence,

$$
\begin{aligned}
& \left(\sum_{j=1}^{q} \omega(j)-M-1\right) d \operatorname{deg}(f) \\
& \quad \leq \sum_{j=1}^{q} \sum_{P \notin E} \omega(j) \frac{d}{d_{j}} \min \left\{M, v_{Q_{j}(f)}(P)\right\}+\frac{1}{2} M(M+1)\{2(g-1)+|E|\} .
\end{aligned}
$$

By using the analogous arguments as in Step 1, we get

$$
\begin{aligned}
(q & \left.-\frac{(2 N-n+1)(M+1)}{n+1}\right) \operatorname{deg}(f) \\
& \leq \sum_{j=1}^{q} \sum_{P \notin E} \frac{1}{d_{j}} \min \left\{M, v_{Q_{j}(f)}(P)\right\}+\frac{(2 N-n+1) M(M+1)}{2(n+1)} \cdot \frac{2(g-1)+|E|}{d} .
\end{aligned}
$$

Case 2: the general case

Assume that $f: S \rightarrow \mathbb{P}^{n}(\mathbb{C})$ is a holomorphic curve such that $f(S) \subset \mathbb{P}^{k}(\mathbb{C})$ and $f(S)$ is not contained in any hypersurface in $\mathbb{P}^{k}(\mathbb{C})$ for some $1 \leq k<n$. It follows that $F: S \rightarrow \mathbb{P}^{M}(\mathbb{C})$ is linearly non-degenerate, where

$$
M=\left(\begin{array}{c}
k+d \\
k
\end{array}\right)-1
$$


Since $Q_{1}, \ldots, Q_{q}$ are in $N$-subgeneral position in $\mathbb{P}^{n}(\mathbb{C})$, their restrictions to $\mathbb{P}^{k}(\mathbb{C})$, $Q_{1} \cap \mathbb{P}^{k}(\mathbb{C}), \ldots, Q_{q} \cap \mathbb{P}^{k}(\mathbb{C})$ are in $N$-subgeneral position in $\mathbb{P}^{k}(\mathbb{C})$. For simplicity, we still denote $Q_{j} \cap \mathbb{P}^{k}(\mathbb{C})$ as $Q_{j}(1 \leq j \leq q)$. Applying Case 1 for $f$ and for the hypersurfaces $\left\{Q_{i}\right\}_{i=1}^{q}$, we have

$$
\begin{aligned}
(q & \left.-\frac{(2 N-k+1)(M+1)}{k+1}\right) \operatorname{deg}(f) \\
& \leq \sum_{j=1}^{q} \sum_{P \notin E} \frac{1}{d_{j}} \min \left\{M, v_{Q_{j}(f)}(P)\right\}+\frac{(2 N-k+1) M(M+1)}{2(k+1)} \cdot \frac{2(g-1)+|E|}{d} .
\end{aligned}
$$

The proof of Theorem 1 is complete.

\section{The proof of Theorem 2}

Since $S$ is an algebraic minimal surface, $S$ has finite total curvature (see [2]). It follows that $S$ is conformally equivalent to a compact surface $\bar{S}$ punctured at a finite number of points $P_{1}, \ldots, P_{r}$ and the generalized Gauss map $G$ extends holomorphically to $\bar{G}: \bar{S} \rightarrow \mathbb{P}^{m-1}(\mathbb{C})$. Let

$$
\left\{Q_{1}, \ldots, Q_{r_{0}}, Q_{r_{0}+1}, \ldots, Q_{q}\right\}
$$

be the set of totally ramified hypersurfaces of $\bar{G}$, located in $N$-subgeneral position, where $Q_{r_{0}+1}, \ldots, Q_{q}$ are exceptional hypersurfaces. Put

$$
E=\left\{P_{1}, \ldots, P_{r}\right\}
$$

By the results of S. S. Chern and R. Osserman [2], we have

$$
C(S)=-2 \pi \operatorname{deg}(\bar{G}) \leq 2 \pi(\mathcal{X}-r)=2 \pi(2-2 g-r-r),
$$

where $\mathcal{X}$ is the Euler characteristic of $\bar{S}$. Hence,

$$
2(g-1) \leq \operatorname{deg}(\bar{G})-2 r .
$$

This implies

$$
2(g-1)+|E| \leq \operatorname{deg}(\bar{G})-r<\operatorname{deg}(\bar{G}) .
$$

Applying the second main theorem for algebraic curves $\bar{G}$ with $E$ and using (4.1), we have

$$
\begin{aligned}
(q & \left.-\frac{(2 N-k+1)\left(M_{k}+1\right)}{k+1}\right) \operatorname{deg}(\bar{G}) \\
< & \sum_{j=1}^{r_{0}} \sum_{P \notin E} \frac{1}{d_{j}} \min \left\{M_{k}, v_{Q_{j}(\bar{G})}(P)\right\}+\sum_{j=r_{0}+1}^{q} \sum_{P \notin E} \frac{1}{d_{j}} \min \left\{M_{k}, v_{Q_{j}(\bar{G})}(P)\right\} \\
& +\frac{(2 N-k+1) M_{k}\left(M_{k}+1\right)}{2(k+1)} \cdot \frac{\operatorname{deg} \bar{G}}{d}
\end{aligned}
$$

where

$$
M_{k}=\left(\begin{array}{c}
k+d \\
k
\end{array}\right)-1
$$


Since $Q_{r_{0}+1}, \ldots, Q_{q}$ are exceptional hypersurfaces, for $P \notin E, v_{Q_{j}(f)}(P)=0$ for $r_{0}+1 \leq$ $j \leq q$. On the other hand, for every $P \in S$ and $1 \leq j \leq r_{0}$, we have

$$
\min \left\{M_{k}, v_{Q_{j}(\bar{G})}(P)\right\} \leq M_{k} \cdot \min \left\{1, v_{Q_{j}(\bar{G})}(P)\right\} \leq \frac{M_{k}}{m_{j}} v_{Q_{j}(\bar{G})}(P) .
$$

By Lemma 5, we get

$$
\begin{aligned}
\sum_{j=1}^{r_{0}} \sum_{P \notin E} \frac{M_{k}}{m_{j} d_{j}} v_{Q_{j}(\bar{G})}(P) & \leq \sum_{j=1}^{r_{0}} \sum_{P \in \bar{S}} \frac{M_{k}}{m_{j} d_{j}} v_{Q_{j}(\bar{G})}(P) \\
& =\sum_{j=1}^{r_{0}} \frac{M_{k} d_{j} \operatorname{deg}(\bar{G})}{m_{j} d_{j}} \\
& =\sum_{j=1}^{r_{0}} \frac{M_{k} \operatorname{deg}(\bar{G})}{m_{j}} .
\end{aligned}
$$

Combining this with (4.2) and (4.3), we have

$$
\begin{aligned}
(q & \left.-\frac{(2 N-k+1)\left(M_{k}+1\right)}{k+1}\right) \operatorname{deg}(\bar{G}) \\
& <\sum_{j=1}^{r_{0}} \frac{M_{k} \operatorname{deg}(\bar{G})}{m_{j}}+\frac{(2 N-k+1) M_{k}\left(M_{k}+1\right)}{2(k+1)} \cdot \frac{\operatorname{deg}(\bar{G})}{d} .
\end{aligned}
$$

For all $1 \leq k \leq m-1$, this implies that

$$
\sum_{j=1}^{q}\left(1-\frac{M_{k}}{m_{j}}\right) \leq q-\sum_{j=1}^{r_{0}} \frac{M_{k}}{m_{j}}<\frac{(2 N-k+1)\left(M_{k}+1\right)\left(M_{k}+2 d\right)}{2(k+1) d} .
$$

Consider $d=1$. Then $d_{i}=1(1 \leq i \leq q)$, hence $M_{k}=k$. This together with (4.5) implies

$$
\sum_{j=1}^{q}\left(1-\frac{k}{m_{j}}\right)<\frac{(2 N-k+1)(k+2)}{2} .
$$

Since $(2 N-k+1)(k+2) \leq(2 N-m+1)(m+1)$ for $1 \leq k \leq m-1$,

$$
\sum_{j=1}^{q}\left(1-\frac{m-1}{m_{j}}\right)<\frac{(2 N-m+2)(m+1)}{2} .
$$

We now consider $d>1$. For $1 \leq k \leq m-1$, we put

$$
A_{k}=(2 N-k+1) \frac{M_{k}+1}{k+1}=(2 N-k+1) \frac{a_{k}}{k+1},
$$

where

$$
a_{k}=\left(\begin{array}{c}
k+d \\
k
\end{array}\right)
$$

We claim that

$$
A_{k} \geq A_{k-1}, \quad 1<k \leq m-1 .
$$


In fact, we have

$$
\begin{aligned}
A_{k} & =\frac{2 N-k+1}{k+1} \cdot \frac{k+d}{k} a_{k-1} \\
& =(2 N-k+1)\left(\frac{a_{k-1}}{k}+\frac{d-1}{k+1} \cdot \frac{a_{k-1}}{k}\right) \\
& =(2 N-k+2) \frac{a_{k-1}}{k}+\frac{a_{k-1}}{k}\left[\frac{d-1}{k+1}(2 N-k+1)-1\right] \\
& \geq A_{k-1} .
\end{aligned}
$$

This yields the claim.

For each $1 \leq k \leq m-1$, set

$$
B_{k}=\frac{(2 N-k+1)\left(M_{k}+1\right)\left(M_{k}+2 d\right)}{2(k+1) d}=\frac{A_{k}\left(M_{k}+2 d\right)}{2 d} .
$$

By the claim, we have

$$
B_{k+1}=\frac{A_{k+1}\left(M_{k+1}+2 d\right)}{2 d}>\frac{A_{k}\left(M_{k}+2 d\right)}{2 d}=B_{k} .
$$

Hence $B_{k}<B_{m-1}, 1 \leq k<m-1$. This together with (4.5) implies that

$$
\sum_{j=1}^{q}\left(1-\frac{M}{m_{j}}\right)<B_{m-1}=\frac{(2 N-m+2)(M+1)(M+2 d)}{2 d m},
$$

where

$$
M=M_{m-1}=\left(\begin{array}{c}
m-1+d \\
m-1
\end{array}\right)-1 .
$$

We finish the proof of Theorem 2 .

Acknowledgements. This work was done during a stay of the authors at the Vietnam Institute for Advanced Study in Mathematics (VIASM). We would like to thank VIASM for partial support, and the staff of VIASM for their hospitality. We also would like to express our gratitude to the referee. His/her valuable comments made on the first version of this paper led to significant improvements in the paper.

\section{REFERENCES}

[1] D. P. An, S. D. Quang and D. D. Thai. The second main theorem for meromorphic mappings into a complex projective space. Acta Math. Vietnam. 38(1) (2013), 187-205.

[2] S. S. Chern and R. Osserman. Complete minimal surface in euclidean $n$-space. J. Anal. Math. 19 (1967), $15-34$.

[3] P. Griffiths and J. Harris. Principles of Algebraic Geometry. Wiley, New York, 1994.

[4] L. Jin and M. Ru. Algebraic curves and the Gauss map of algebraic minimal surfaces. Differ. Geom. Appl. 25 (2007), 701-712.

[5] Y. Kawakami. On the totally ramified value number for the Gauss map of minimal surfaces. Proc. Japan Acad. Ser. A Math. Sci. 82 (2006), 1-3.

[6] Y. Kawakami, R. Kobayashi, and R. Miyaoka. The Gauss map of pseudo-algebraic minimal surfaces. Forum Math. 20 (2008), 1055-1069, doi:10.1515/FORUM.2008.047. 
[7] M. Ru. On the Gauss map of minimal surfaces immersed in $\mathbb{R}^{n}$. J. Differential Geom. 34 (1991), 411-423.

[8] D. D. Thai and P. D. Thoan. The Gauss map of algebraic complete minimal surfaces omits hypersurfaces in subgeneral position. Vietnam J. Math. (2017), doi:10.1007/s10013-017-0259-6.

Do Duc Thai

Department of Mathematics

Hanoi National University of Education

136 Xuan Thuy str.

Hanoi

Vietnam

(E-mail:doducthai@hnue.edu.vn)

Pham Duc Thoan

Department of Mathematics

National University of Civil Engineering

55 Giai Phong str.

Hanoi

Vietnam

(E-mail: ducthoan.pham@gmail.com) 